\title{
REMARKS ON A PAPER OF HERMANN
}

\author{
BY \\ VICTOR W. GUILLEMIN AND SHLOMO STERNBERG
}

Let $G$ be a Lie group acting differentiably on a manifold and let $p$ be a point left fixed by $G$. If $G$ is compact, a well-known result of $\mathrm{H}$. Cartan asserts that the action of $G$ in a neighborhood of $p$ is equivalent to the linear action of $G$ on the tangent space at $p$, cf. [4]. Palais and Smale have suggested extending this theorem to noncompact Lie groups. Hermann, in the preceding paper has shown that the corresponding formal problem can be reduced to a cohomology question which can always be solved if the group is semisimple (cf. the discussion of this point in $\$ 1$ below). In this paper we will concentrate on the semisimple case.

If the group $G$ is connected, then instead of trying to find a linear system of coordinates for $G$, one can try to find a linear system of coordinates for the vector fields corresponding to the one-parameter subgroups of $G$. More generally one can just consider a representation of a semisimple Lie algebra as an algebra of vector fields on a manifold, and try to determine the local behavior in the neighborhood of a common zero of all the vector fields. Hermann [2] proved that with the hypotheses above and $C^{\infty}$ or analytic data one can always find a formal power series change of coordinates which formally linearizes all the vector fields at the critical point. However, it seems quite difficult to get a convergence proof by Hermann's method except in the relatively few cases where the power series change of coordinates is unique. In this paper we use àn alternate approach based on complexification and the Weyl unitary trick to prove a linearization theorem if the data are analytic. We will also show that the analogous theorem is false in the $C^{\infty}$ case unless some restrictions are placed on the algebra. What restrictions is unclear at present, but it seems that the algebra $\operatorname{sl}(2, R)$ has to be singled out for special consideration.

1. Let $g$ be a finite dimensional Lie algebra and $\sim: g \rightarrow L_{\text {analytic }}$, a representation of $g$ in the algebra of real (or complex) analytic vector fields on a real (or complex) analytic manifold, $M$. Let $p$ be a common fixed point of all the vector fields in the image, $\tilde{g}$. We will establish a simple criterion for all the vector fields in $\tilde{g}$ to be simultaneously linearizable in a neighborhood of $p$.

Proposition 1.1. A necessary and sufficient condition for the representation $\sim: g \rightarrow L_{\text {analytic, }}$ to be locally equivalent, via an analytic diffeomorphism, to a linear representation of $g$, is that there exist an analytic vector field, $X$, defined in $a$

Received by the editors November 1, 1966. 
neighborhood of $p$, vanishing at p, having the identity matrix as its Jacobian at $p$ and commuting with all the vector fields in $\tilde{g}$.

Proof. Assume such a vector field $X$ exists. By a fundamental theorem of Poincaré [4] there exists an analytic system of coordinates $\left(x_{1}, x_{2}, \ldots, x_{n}\right)$, with origin at $p$ and with

$$
X=x_{1}\left(\partial / \partial x_{1}\right)+\cdots+x_{n}\left(\partial / \partial x_{n}\right)
$$

Let $Y$ be any vector field that commutes with $X$. We will show that $Y$ is linear in the above coordinates. Writing $Y$ in its power series expansion about the origin: $Y=\sum Y^{k}$, and bracketing $Y$ with $X$ we get

$$
[X, Y]=\sum k Y^{k}=0
$$

by Euler's identity. Therefore, $Y^{k}=0$ for $k \neq 0$. It follows from this and the hypotheses about $X$ that the system of coordinates introduced above linearizes each of the vector fields in $\tilde{g}$.

To prove the converse, suppose there exists a system of coordinates $\left(x_{1}, x_{2}, \ldots, x_{n}\right)$ with origin at $p$, in which the vector fields belonging to $\tilde{g}$ are linear. Let $X$ be defined by the expression (1.1). Q.E.D. (The corresponding formal proposition is equally valid.)

As an application of Proposition 1.1 we will produce some examples of representations of Lie algebras which cannot be linearized. Let $V$ be a finite-dimensional vector space over the real or complex numbers, and let $V^{*}$ be its dual space. By standard identifications we can identify $S^{k}\left(V^{*}\right)$ with homogeneous polynomials of degree $k$ in $V$, and we can identify $V \otimes S^{k}\left(V^{*}\right)$ with vector fields on $V$ having homogeneous polynomials of degree $k$ as coefficients. Let $g$ be a finite-dimensional Lie algebra, and let

$$
\rho: g \rightarrow \sum_{k=1}^{\infty} V \otimes S^{k}\left(V^{*}\right)
$$

be a homomorphism of $g$ into the algebra of polynomial vector fields on $V$ vanishing at 0 . Let $\rho_{k}: g \rightarrow V \otimes S^{k}\left(V^{*}\right)$ be the $k$ th component of $\rho$. By identifying $V \otimes V^{*}$ with Hom $(V)$, one can easily see that $\rho_{1}$ defines a homomorphism of $g$ into Hom $(V)$ or in other words a representation of $g$ on $V$. This induces representations of $g$ on $V^{*}$ and $V \otimes S^{k}\left(V^{*}\right)$. Therefore, one can define cohomology groups, $H^{i}\left(g, V \otimes S^{k}\left(V^{*}\right)\right)$ for all $i$ and $k$ (cf. Jacobson [3] for details about cohomology of Lie algebras).

Proposition 1.2. The mapping $\rho_{2}: g \rightarrow V \otimes S^{2}\left(V^{*}\right)$ defines a comohology class in $H^{1}\left(g, V \otimes S^{2}\left(V^{*}\right)\right)$. If the representation $\rho$ can be linearized, this cohomology class vanishes. 
Proof. Let $\alpha$ and $\beta$ be elements of $g$. Since $\rho$ is an algebra homomorphism, we get:

$$
\left[\rho_{1}(\alpha), \rho_{2}(\beta)\right]-\left[\rho_{1}(\beta), \rho_{2}(\alpha)\right]=\rho_{2}([\alpha, \beta]),
$$

which is exactly the statement that $\rho_{2}$ defines a one-cycle in the complex associated with $\left(g, V \otimes S^{2}\left(V^{*}\right)\right)$. If the representation can be linearized there exists a vector field $Z$ which vanishes at the origin, has the identity as its Jacobian at the origin, and commutes with all $\rho(\dot{\alpha})$. If $Z_{1}$ and $Z_{2}$ are the first two terms in the power series expansion of $Z$ about the origin we get:

$$
\left[\rho_{1}(\alpha), Z_{2}\right]+\left[\rho_{2}(\alpha), Z_{1}\right]=0,
$$

and since $Z_{1}$ is the identity, we get:

$$
\left[\rho_{1}(\alpha), Z_{2}\right]=\rho_{2}(\alpha),
$$

which, when reinterpreted as a statement about the cohomology, says simply that $\rho_{2}$ is a coboundary.

By induction, if $H^{1}\left(g, V \otimes S^{k}\left(V^{*}\right)\right)=0$ for all $k$ then $\rho$ can be linearized.

COROLlARY. If $H^{1}\left(g, V^{*}\right) \neq 0$, there exists a representation

$$
g \rightarrow \sum_{k=1,2} V \otimes S^{k}\left(V^{*}\right)
$$

which is not equivalent, via an analytic diffeomorphism to a linear representation of $g$.

Proof. Let $I$ be the identity mapping in Hom $(V)$. We can think of this as an element of $V \otimes V^{*}$. By tensoring elements of $V^{*}$ with $I$ and then symmetrizing on the last two terms, we get a mapping:

$$
\tau: V^{*} \rightarrow V \otimes S^{2}\left(V^{*}\right)
$$

Also there is a contraction mapping:

$$
\tau^{\prime}: V \otimes S^{2}\left(V^{*}\right) \rightarrow V,
$$

and it is easy to see that the composition of $\tau$ and $\tau^{\prime}$ is a mapping of $V$ onto itself which is a multiple of the identity mapping. Thus the induced mapping:

$$
\tau_{*}: H^{1}\left(g, V^{*}\right) \rightarrow H^{1}\left(g, V \otimes S^{2}\left(V^{*}\right)\right)
$$

is injective.

Now let $\lambda$ be a cocycle in Hom $\left(g, V^{*}\right)$ which projects onto a nonzero cohomology class in $H^{1}\left(g, V^{*}\right)$ and let $\rho_{1}=\tau \circ \lambda$. It is easy to see that the mapping:

$$
\rho_{0}+\rho_{1}: g \rightarrow \sum_{k=1,2} V \otimes S^{k}\left(V^{*}\right)
$$

is an algebra homomorphism, and by Proposition 1.2 it is not locally equivalent, via an analytic diffeomorphism, to a linear representation. 
REMARK 1. With a little more work one can show that the representation (1.3) is not even $C^{2}$ equivalent to a linear representation.

RemarK 2. As is well known $H^{1}\left(g, V^{*}\right)=0$ for all representation spaces $V$ providing $g$ is semisimple. On the other hand, if $g$ is not semisimple, one can always find a representation space $V$ for which $H^{1}\left(g, V^{*}\right) \neq 0$.

REMARK 3. What can be concluded about nonsemisimple algebras from Remark 2 applies equally well to simply connected nonsemisimple groups, since the representation (1.3) can be integrated to give a representation of the group associated with $g$ as collineations on the projective completion of $V$.

2. Let $g$ be a real semisimple Lie algebra and let $\sim: g \rightarrow L_{\text {analytic }}$ be a homomorphism of $g$ into the algebra of real analytic vector fields on a real analytic manifold, $M$. Let $p$ be a point at which the vector fields in the image, $\tilde{g}$, have a common zero. In this section we will show that one can find an analytic system of coordinates, centered at $p$, in which all of the vector fields in $\tilde{g}$ are linear. To do this let us choose any analytic system of coordinates centered at $p$. Since we can restrict our attention to one coordinate patch, we can think of $M$ as a small disk about the origin in $R^{n}$ with the origin being the point $p$. If we choose this disk sufficiently small, the vector fields in $\tilde{g}$ can be analytically extended to a small disk $D$ about the origin in $C^{n}$, and we get a representation of $g$ by holomorphic vector fields on $D$. This can be extended to a representation of the complex semisimple Lie algebra, $g_{R} \otimes C$ (by letting $\left.(\sqrt{ }-1 x)^{\sim}=(\sqrt{ }-1 \tilde{x})\right)$. Let $h$ be a compact real form for $g_{R} \otimes C$. We also get a representation of $h$ as a Lie algebra of holomorphic vector fields on $D$. We will continue to denote this by $\sim$.

Let $D^{\prime}$ be a closed disk centered at the origin and containing $D$. Let $f$ be a holomorphic homeomorphism of $D^{\prime}$ into $C^{n}$ which maps the origin into the origin. By Schwarz's lemma:

$$
|f(z)| \leqq|z|\left(1 / R_{D^{\prime}}\right)|f|_{\text {sup }}, \quad z \in D^{\prime},
$$

where $R_{D^{\prime}}$ is the radius of $D^{\prime}$ and $|f|_{\text {sup }}$ is the supremum of $f$ over $D^{\prime}$. Let $G$ be the set of germs of holomorphic diffeomorphisms of $C^{n}$ into $C^{n}$ with source and target at the origin. It is clear that $G$ has the structure of an abstract group. Let $H$ be the compact simply-connected Lie group associated with the Lie algebra $h$. Assume a metric | | is defined on $h$.

Proposition 2.1. There exist an $\varepsilon>0$ and a disk $D^{\prime}$, centered about the origin and contained in $D$, such that if $|x|<\varepsilon, \exp \tilde{x}$ is defined on all of $D^{\prime}$. There exists a constant, $K$, such that

$$
|\exp \tilde{x}|_{\text {sup }}<K e^{|x|}
$$

for all $x$ in $h$ of norm less than $\varepsilon$ (the supremum on the left being over $\left.D^{\prime}\right)$. The mapping: $\exp x \rightarrow \exp \tilde{x}$ is unambiguously defined for all $x$ of norm $<\varepsilon$ in $h$ and 
defines a homomorphism $\left(^{1}\right)$ of a neighborhood, $N$, of the identity in $H$ into $G$. Moreover, the mapping, $N \times D^{\prime} \rightarrow C^{n}$, is real-analytic in the first factor and complexanalytic in the second.

Proposition 2.1 is a standard result if the vector fields, $\tilde{x}$, are real and $C^{\infty}$. With slight modifications the same proof works when the $\tilde{x}$ are holomorphic.

COROLlARY. There exists a group homomorphism, $\rho: H \rightarrow G$, extending the local homomorphism described in Proposition 2.1.

For the proof, which is based on the simple-connectedness of $H$, see [1, Chapter II, §VIII, Theorem 3]( $\left.{ }^{2}\right)$.

We will show that there exists a fixed disk $D^{\prime}$, to which the germs of mappings in $G$ which come from $H$ can be simultaneously extended. To do this we will need the following lemma.

LEMMA. If $H$ is a connected group and $N$ is a neighborhood of the origin in $H$, there exists a positive integer, $k$, such that every element in $H$ is of the form $a_{1} a_{2} \cdots a_{k}$ where $a_{i}$ is in $N$ for $i=1,2, \ldots, k$.

Proof. Consider all right translates of $N$ by products of the form indicated. By connectedness these sets must cover $G$, and by compactness there is a finite subcovering.

Proposition 2.2. There exists a disk $D^{\prime \prime}$, centered at the origin and contained in $D^{\prime}$, such that the mapping $N \times D^{\prime} \rightarrow C^{n}$ defined in Proposition 2.1 can be extended to a mapping $H \times D^{\prime \prime} \rightarrow C^{n}$, which induces the homomorphism $\rho: H \rightarrow G$. Moreover, this mapping is real-analytic in the first factor and holomorphic in the second.

Proof. By Proposition 2.1 every element in $N$ corresponds to a mapping $f$ of $D^{\prime}$ into $C^{n}$ which is bounded by

$$
|f|_{\text {sup }}<K e^{\varepsilon} \text {. }
$$

By Equation 2.1 every such $f$ satisfies the inequality

$$
|f(z)|<|z| \frac{1}{R_{D^{\prime}}} K e^{\varepsilon}=A|z|, \quad \text { where } A=\frac{1}{R_{D^{\prime}}} K e^{\varepsilon} .
$$

Let $D^{\prime \prime}$ be the disk with center about the origin and radius $R_{D^{\prime}} / A^{k}$, where $k$ is an integer satisfying the hypotheses of the lemma. If $f_{1}, f_{2}, \ldots, f_{k}$ are $k$ mappings of $D^{\prime}$ into $C^{n}$ corresponding to elements of $N$, their composition, $f_{1} \circ f_{2} \circ \cdots \circ f_{k}$, is defined on $D^{\prime \prime}$. Thus by the lemma every element of $G$ which is in the image of $H$ comes from a mapping with domain $D^{\prime \prime}$. The analytic dependence on $H$ follows from Proposition 2.1. Q.E.D.

(1) This is, of course, in the sense of local groups: $\exp x \cdot \exp y \rightarrow \exp \tilde{x} \cdot \exp \tilde{y}$ whenever both terms on the left are in $N$ and their product is in $N$.

${ }^{2}$ In this theorem of Chevalley no topology is needed on $G$. 
Given an element $a$ in $H$, let us denote by $T_{a}$ the corresponding mapping of $D^{\prime \prime}$ into $C^{n}$. We will also consider the following holomorphic vector field defined on all of $C^{n}$ :

$$
X_{0}=z_{1} \frac{\partial}{\partial z_{1}}+z_{2} \frac{\partial}{\partial z_{2}}+\cdots+z_{n} \frac{\partial}{\partial z_{n}}
$$

If $q$ is a point of $D^{\prime \prime},\left(T_{a}\right)_{*} X_{0}$ is defined at $q$, is real-analytic as a function of $a$, and is holomorphic as a function of $q$. Define

$$
X=\int_{H}\left(T_{a}\right)_{*} X_{0} d a,
$$

where $d a$ is Haar measure on the group $H . X$ is a holomorphic vector field defined on all of $D^{\prime \prime}$. By construction it is invariant under the local action of $H$; therefore it commutes with all the vector fields in $\tilde{h}$. Obviously it also commutes with all complex linear combinations of these vector fields. Let $A$ be the Jacobian matrix of $T_{a}$ at the origin. $X_{0}$ vanishes at the origin, and its Jacobian is the identity matrix $I$; therefore the Jacobian of $\left(T_{a}\right)^{*} X_{0}=A I A^{-1}=I$. It follows that the integrand of (2.4) is identically $I$ when $q=0$; therefore, if the Haar measure of the group is normalized so that the measure of the whole group is 1 , the vector field $X$ vanishes at the origin and has the identity matrix as its Jacobian at the origin. To conclude the proof we need the following lemma which ensures that if we go back from $C^{n}$ to $R^{n}$ the relations above are preserved.

Lemma. Let $D$ be a disk centered at the origin in $C^{n}$, and let $D_{0}=D \cap R^{n}$. If $f$ is a holomorphic function defined on $D$, let $\hat{f}$ be its restriction to $D_{0}$, and if

$$
Z=\sum a_{i}\left(z_{1}, z_{2}, \ldots, z_{n}\right)\left(\partial / \partial z_{i}\right)
$$

is a holomorphic vector field defined on $D$, let $\hat{Z}=\hat{a}_{i}\left(\partial / \partial x_{i}\right)$. Then $(\hat{Z})(\hat{f})=(Z f)^{\wedge}$ and $\left(\left[Z_{1}, Z_{2}\right]^{\wedge}\right)=\left[\hat{Z}_{1}, \hat{Z}_{2}\right]$.

Proof. The first identity follows from the Cauchy-Riemann equations, and the second identity from the first by showing that both sides are the same when evaluated on functions of the form $\hat{f}$.

Applying this lemma to the vector field, $X$, defined by Equation (2.4), we get a vector field $\hat{X}$ defined on $D^{\prime \prime} \cap R^{n}$ which commutes with all the vector fields in $\tilde{g}$ and has the Taylor series development:

$$
X=\sum x_{i} \frac{\partial}{\partial x_{i}}+O\left(|x|^{2}\right)
$$

at the origin. All the vector fields in $\tilde{g}$ are real-valued; so $\operatorname{Re} \tilde{X}$ also commutes with $\tilde{g}$ and, of course, still has the Taylor series development (2.5) at the origin. Applying Proposition 1.1 we conclude that the representation $\sim$ can be linearized. 
3. We will give an example to show that the theorem proved in $\$ 2$ is not true for $C^{\infty}$ representations of semisimple algebras. It is still possible that the theorem may be true for $C^{\infty}$ representations of semisimple groups, and also for a suitably restricted class of semisimple algebras.

Consider the following vector fields in $R^{3}$ :

$$
x \frac{\partial}{\partial y}-y \frac{\partial}{\partial x}, x \frac{\partial}{\partial z}+z \frac{\partial}{\partial x}, y \frac{\partial}{\partial z}+z \frac{\partial}{\partial y} .
$$

One can easily see that (3.1) is the representation of $\operatorname{sl}(2, R)$ as the three dimensional Lorentz algebra which leaves fixed the bilinear form: $x^{2}-y^{2}-z^{2}$ $=r^{2}-z^{2}$. Let $g(u)$ be a $C^{\infty}$ function of one variable which is identically zero on the negative real axis, and consider the following vector fields:

$$
\begin{gathered}
x \frac{\partial}{\partial y}-y \frac{\partial}{\partial x}+g\left(r^{2}-z^{2}\right)\left(x \frac{\partial}{\partial x}+y \frac{\partial}{\partial y}+z \frac{\partial}{\partial z}\right), \\
x \frac{\partial}{\partial z}+z \frac{\partial}{\partial x}-\frac{y z}{r^{2}} g\left(r^{2}-z^{2}\right)\left(x \frac{\partial}{\partial x}+y \frac{\partial}{\partial y}+z \frac{\partial}{\partial z}\right), \\
y \frac{\partial}{\partial z}+z \frac{\partial}{\partial y}+\frac{x z}{r^{2}} g\left(r^{2}-z^{2}\right)\left(x \frac{\partial}{\partial x}+y \frac{\partial}{\partial y}+z \frac{\partial}{\partial z}\right) .
\end{gathered}
$$

It is easy to show that these vector fields are $C^{\infty}$ everywhere and satisfy the same bracket relations as (3.1). Inside the cone $r^{2}-z^{2}$ the two sets of vector fields are identical; however, if we choose $g(u)=e^{-1 / u^{2}}, u>0$, and $g(u)=0, u \leqq 0$, for example, then outside the cone $r^{2}-z^{2}=0$, the vector field $x(\partial / \partial y)-y(\partial / \partial x)$ has closed circular orbits while the corresponding vector field in (3.1)' has orbits which spiral outward; so it is impossible to find a $C^{\infty}$ mapping, defined in a neighborhood of the origin and carrying (3.1) into (3.1)'.

\section{BIBLIOGRAPHY}

1. Claude Chevalley, Theory of Lie groups. I, Princeton Math. Series, Vol. 8, Princeton Univ. Press, Princeton, N. J., 1946.

2. Robert Hermann, The formal linearization of a semisimple Lie algebra of vector fields about a singular point, Trans. Amer. Math. Soc. 130 (1968), 105-109.

3. Nathan Jacobson, Lie algebras, Interscience, New York, 1961.

4. Henri Poincaré, Oeuvres, Vol. 1, pp. 95-114, Gauthier-Villars, Paris, 1951.

HARVARD UNIVERSITY,

Cambridge, Massachusetts

Massachusetts Institute of TeChNology,

Cambridge, Massachusetts 